\title{
Prognostic utility of basaloid differentiation in oropharyngeal cancer
}

Timothy Cooper ${ }^{1}$, Vincent Biron ${ }^{1}$, Ben Adam², Alexander C Klimowicz ${ }^{3}$, Lakshmi Puttagunta ${ }^{2}$ and Hadi Seikaly ${ }^{*}$

\begin{abstract}
Background: Human papillomavirus (HPV) is recognized as the key risk factor for a distinct subset of oropharyngeal squamous cell carcinoma. P16 is a reliable, sensitive surrogate marker for HPV and confers a positive prognostic advantage. Basaloid differentiation on hematoxylin and eosin (H\&E) staining is anecdotally noted by some pathologists to be associated with p16 positivity. This association, however, has not been adequately quantified in the literature, nor has the prognostic implications of basaloid differentiation been described.
\end{abstract}

Objectives: 1) To correlate the H\&E staining feature of basaloid differentiation with p16 positivity in oropharyngeal cancer. 2) To investigate the prognostic utility of basaloid differentiation in oropharyngeal cancer survival.

Methods: Retrospective cross-sectional study of all patients diagnosed with and treated for oropharyngeal cancer at a single tertiary cancer center from 2002 to 2009. Tissue microarrays (TMAs) were generated from 208 oropharyngeal tumor specimens stained with H\&E and immunohistochemical markers. These oropharyngeal TMAs were utilized in several previous publications. Samples were scored for basaloid differentiation by a pathologist blinded to the p16 result. A multivariate survival analysis with Cox-regression and Kaplan-Meier survival analysis was performed.

Results: In the 208 samples, basaloid differentiation correlated with p16 positivity (Spearman's rho 0.435). Basaloid differentiation and p16 positivity were both independent predictors of improved survival. The 5 year disease specific survival (DSS) was 73\% for p16 positive tumors and 35\% for p16 negative tumors $(p<0.001)$. Similarly, the 5 year DSS of basaloid differentiated tumors was $74 \%$ compared to $41 \%$ for non-basaloid tumors $(p=0.001)$. Patients with $p 16$ positive and basaloid differentiated tumors had the best survival outcomes with a 5 year DSS of 80\%.

Conclusions: Basaloid differentiation is a feature on H\&E which correlates with p16 positivity and is a simple, inexpensive, independent, positive prognostic indicator of comparable magnitude to p16 status. Due to the added prognostic value of basaloid differentiation, this feature should be routinely reported by qualified pathologists.

Keywords: Basaloid differentiation, HPV, p16, Hematoxylin, Eosin, Oropharynx, Squamous cell carcinoma, Outcomes, Survival

\section{Background}

Human papillomavirus (HPV) is recognized as the key risk factor for a distinct subset of oropharyngeal squamous cell carcinoma [1-4]. The proportion of oropharyngeal cancer attributable to HPV is increasing dramatically and is now thought to account for approximately $70 \%$ of oropharyngeal squamous cell carcinoma $[1,5-7]$.

\footnotetext{
* Correspondence: hadi.seikaly@albertahealthservices.ca

'Division of Otolaryngology - Head and Neck Surgery, Department of Surgery, University of Alberta, 1 E4 University of Alberta Hospital, Edmonton, AB T6G 2B7, Canada

Full list of author information is available at the end of the article
}

There are numerous assays for the detection of HPV in tumor cells. These include immunohistochemistry (IHC) for p16 protein, polymerase chain reaction (PCR) and in-situ hybridization techniques for detection of viral DNA, and reverse transcriptase PCR (RT-PCR) for viral mRNA [8,9]. The gold standard for HPV detection is RT-PCR for viral E6 and E7 mRNA, although it is not routinely performed [9]. Commonly, p16 IHC is performed [10]. P16 is a cyclin-dependent kinase inhibitor which is overexpressed in cells infected with HPV $[11,12]$. Studies have shown that p16 IHC is a reliable, sensitive surrogate marker for HPV and confers a positive prognostic advantage $[6,8,10,13]$.

\section{Biomed Central}


Hematoxylin and eosin (H\&E) staining is routinely performed on all biopsy and surgical specimens submitted for pathology. It is an inexpensive stain with readily available results. The classical description of HPV-related oropharyngeal cancer histology is non-keratinizing and basaloid differentiated $[3,5,9,13,14]$. Keratinization is the feature that has been focused on in the literature to date and in pathology reports. Basaloid differentiation is anecdotally noted by some pathologists to be associated with p16 positivity. This association, however, has not been adequately quantified in the literature $[3,11,12,15]$, nor has the prognostic implications of basaloid differentiation been described.

The purpose of this study was twofold:

1) to quantify the association of the H\&E marker of basaloid differentiation with p16 IHC in oropharyngeal squamous cell carcinoma.

2) to investigate the prognostic utility of the $H \& E$ marker of basaloid differentiation in oropharyngeal squamous cell carcinoma.

\section{Methods}

This is a retrospective cross-sectional study set in a regional head and neck cancer treatment center. Approval was obtained from the University of Alberta Health Research Ethics Board prior to the commencement of the study. Patients were identified through the Alberta Cancer Registry in a prospective manner from 2002 to 2009 for inclusion in the study. Patient demographics, staging, treatment, and survival data were collected.

All patients diagnosed and treated with oropharyngeal squamous cell carcinoma in Edmonton, Alberta between 2002 and 2009 were eligible for inclusion. Each patient required a core or tissue biopsy to be performed for use in a tissue microarray (TMA). Included patients were treated with curative intent with any combination of cancer treatment modalities including surgery, chemotherapy, and radiation.

Patients and their associated TMAs were excluded if their cancer was treated with palliative intent or inadequate tissue was obtained for assessment of H\&E staining features or determination of p16 status.

\section{TMA construction}

TMAs were constructed with formalin-fixed paraffinembedded (FFPE) tumor tissue from either pre-treatment biopsies or primary surgery. A pathologist reviewed the blocks and excluded cases with inadequate tissue for future diagnosis. FFPE blocks were marked by a pathologist for TMA construction. The TMAs were constructed with duplicate or triplicate cores of FFPE blocks as per the TMA protocol described by Klimowicz et al. [16]. These
TMAs had been utilized in previous studies conducted by the authors.

\section{Immunohistochemistry}

IHC for p16 was performed using the diaminobenzidine (DAB) staining method as previously reported by Lau et al. [10]. In accordance with previously established standards in the literature, p16 positivity was defined as high intensity staining in greater than $70 \%$ of cells scored manually by a pathologist.

\section{Histologic analysis}

H\&E features of each TMA, including basaloid differentiation and keratinization, were scored by a pathology resident and confirmed by a staff head and neck pathologist. Both individuals interpreting the H\&E staining features were blinded to the p16 status of the TMAs. Basaloid differentiation was defined as the presence of two of three features associated with basaloid differentiation that were identifiable on TMA including peripheral palisading, high nuclear to cytoplasmic ratio, and solid growth pattern.

\section{Statistical analysis}

Spearman's correlation was used to calculate the correlation between p16 status and basaloid differentiation. Multivariate analysis was performed using Cox proportional hazards regression for the variables basaloid differentiation, p16 status, age, gender, and treatment modality. Kaplan-Meier survival analysis was performed to calculate disease specific survival (DSS) within subgroups based on histologic features and IHC staining. Comparison of proportions was performed using $X^{2}$ or Fisher's exact test as appropriate and continuous data using Student's $t$-test. Statistical significance was accepted as $\mathrm{P}<0.05$ in all cases.

Statistical analysis was performed using SPSS Version 21 (IBM). The database was initially constructed using Excel 2010 (Microsoft) and converted to SPSS in order for data analysis to be performed.

\section{Results}

A total of 208 patients and their TMAs were included in the study. The mean age was 58.4 years (range 3295 years). There was a male predominance with 161 men and 47 women. 189 patients presented with advanced stage disease (stage 3 or 4) compared to only 19 with early stage disease (stage 1 and 2). Nodal disease was present in 172 patients on presentation. Surgery followed by chemotherapy and radiation was the most common treatment. Of the 208 tumor specimens, 111 (53\%) were p16 positive and 97 (47\%) were p16 negative. Eighty-four (40\%) demonstrated basaloid differentiation while 124 (60\%) were non-basaloid. A breakdown of demographic information as well as staging, tumor characteristics, and treatment modalities used is presented in Table 1 . 


\begin{tabular}{|c|c|c|c|c|c|c|c|}
\hline Characteristic & All & B & NB & $\mathbf{P}$ & $+\mathrm{P} 16$ & -P16 & $\mathbf{P}$ \\
\hline $\mathrm{n}$ & 208 & 84 & 124 & - & 111 & 97 & - \\
\hline Mean age & 58.4 & 57.4 & 59.1 & 0.28 & 55.3 & 62.0 & $<0.01$ \\
\hline Gender (male) & 161 & 64 & 97 & 0.73 & 88 & 73 & 0.49 \\
\hline \multicolumn{8}{|l|}{ P16 Status } \\
\hline Positive & 111 & 69 & 42 & $<0.01$ & - & - & - \\
\hline Negative & 97 & 15 & 82 & & - & - & \\
\hline \multicolumn{8}{|l|}{ Staging (\%) } \\
\hline Advanced & 91 & 94 & 89 & 0.19 & 97 & 84 & $<0.01$ \\
\hline Early & 9 & 6 & 11 & & 3 & 16 & \\
\hline \multicolumn{8}{|l|}{ T Staging (\%) } \\
\hline $\mathrm{T} 1$ & 19 & 23 & 17 & $<0.01$ & 21 & 17 & 0.10 \\
\hline $\mathrm{T} 2$ & 27 & 36 & 21 & & 28 & 26 & \\
\hline T3 & 31 & 33 & 30 & & 35 & 27 & \\
\hline T4 & 23 & 9 & 33 & & 16 & 31 & \\
\hline \multicolumn{8}{|l|}{ N Stage (\%) } \\
\hline NO & 14 & 7 & 18 & 0.04 & 6 & 24 & $<0.01$ \\
\hline $\mathrm{N}$ positive & 86 & 93 & 82 & & 94 & 76 & \\
\hline \multicolumn{8}{|l|}{ Treatment (\%) } \\
\hline Primary surgery & 75 & 77 & 73 & 0.54 & 72 & 68 & 0.06 \\
\hline Primary RT & 25 & 23 & 27 & & 28 & 32 & \\
\hline Surgery + CRT & 37 & 52 & 27 & $<0.01$ & 51 & 21 & $<0.01$ \\
\hline Surgery + RT & 23 & 14 & 28 & & 19 & 27 & \\
\hline CRT & 17 & 17 & 18 & & 14 & 21 & \\
\hline RT & 8 & 6 & 10 & & 5 & 11 & \\
\hline Surgery & 15 & 11 & 18 & & 10 & 21 & \\
\hline
\end{tabular}

$\mathrm{n}=$ number of patients. Primary RT $=$ radiation therapy $+/-$ Chemotherapy. Primary surgery $=$ Surgery $+/-$ radiation therapy $+/-$ Chemotherapy. $\mathrm{B}=$ basaloid differentiated. $\mathrm{NB}=$ non-basaloid. $\mathrm{CRT}=$ chemoradiation. $\mathrm{RT}=$ radiation. $P$ values calculated using $x^{2}$ or Fisher's exact test for categorical data and two tailed t-test for continuous data.

Subgroup analyses comparing patients with or without basaloid differentiation and p16 positivity are also shown (Table 1). Patients who were p16 positive were younger than those who were p16 negative with a mean age of $55.3 \pm 10.1$ years compared to $62.0 \pm 11.2$ years $(\mathrm{p}<0.01)$. There were no significant differences in gender distribution between groups. Patients who had p16 positive tumors were more likely to present with advanced stage disease $(\mathrm{p}<0.01)$ and have positive nodal status $(\mathrm{p}<0.01)$. Basaloid differentiated tumors were more likely to be p16 positive than non-basaloid tumors $(\mathrm{p}<0.01)$. Nonbasaloid differentiated tumors had more advanced $\mathrm{T}$ staging $(\mathrm{p}<0.01)$ while basaloid differentiated tumors were more likely to have positive nodes $(\mathrm{p}=0.04)$, with no significant difference in advanced compared to early stage disease. There were statistically significant differences between groups with regards to the treatment modalities used ( $\mathrm{p}<0.01)$ with $\mathrm{p} 16$ positive and basaloid differentiated patients being more frequently treated with surgery followed by chemoradiation.

Basaloid differentiation correlated with p16 positivity using Spearman's correlation (Table 2), with a correlation coefficient of $0.435(\mathrm{p}<0.001)$. Multivariate survival analysis with Cox proportional hazards regression gave statistically significant values for p16 positivity and basaloid differentiation as independent predictors of survival (Table 3). The calculated hazard ratios were 0.455 for basaloid differentiation, and 1.356 for p16 negativity. Treatment with either chemoradiation or radiation alone were statistically significant predictors of mortality compared to surgery followed by chemoradiation, which was used as a reference $(\mathrm{p}<0.0001)$.

Five year DSS calculated using Kaplan-Meier analysis for p16 positive tumors was $73 \%$ compared to $35 \%$ in p16 negative tumors $(\mathrm{p}<0.001)$ (Figure 1$)$. Similarly, 5 year DSS was $74 \%$ in basaloid differentiated patients compared to $41 \%$ in patients with non-basaloid tumors $(\mathrm{p}=0.001)$ (Figure 2).

By combining basaloid differentiation and p16 status in Kaplan-Meier analysis, DSS could be further stratified (Figure 3). Those patients with basaloid differentiated, p16 positive tumors had a 5 year DSS of $80 \%$, compared to $62 \%$ in non-basaloid, p16 positive patients, $50 \%$ in basaloid differentiated, p16 negative patients, and $32 \%$ in non-basaloid, p16 negative patients $(\mathrm{p}<0.001)$.

\section{Discussion}

Patients in our series who had p16 positive tumors were predominantly male, younger, and had more advanced staged disease with positive nodes; these findings are similar to what others have reported in the literature $[1,17]$. Patients with basaloid differentiated tumors did not have more advanced staging but were more likely to have nodal disease as well. Basaloid differentiation is a feature that has been identified as being associated with HPV-related oropharyngeal cancer $[3,5,9,11,13,14]$. In this study, basaloid differentiation was strongly correlated with p16 positivity but could not consistently predict p16 positivity; as such, it could not replace p16 as a surrogate marker for HPV positivity.

Basaloid differentiation is associated with HPV in oropharyngeal cancer and has been looked at in small numbers by several authors. Laco et al. [15] found that 17 of

Table 2 Spearman's correlation of basaloid differentiation with $\mathbf{p} 16$ positivity

\begin{tabular}{lcc}
\hline Characteristic & $\begin{array}{c}\text { Spearman's rho } \\
\text { correlation coefficient }\end{array}$ & $\mathbf{P}$ \\
\hline Basaloid differentiation & 0.435 & $<0.001$ \\
\hline
\end{tabular}


Table 3 Multivariate survival analysis with Cox proportional hazards regression

\begin{tabular}{lccc}
\hline Variable & HR & $\mathbf{9 5 \% ~ C l}$ & $\mathbf{P}$ \\
\hline Basaloid differentiation & 0.46 & $0.27-0.76$ & 0.003 \\
p16 negative & 1.36 & $1.04-1.77$ & 0.025 \\
Patient variables & & & \\
Male gender & 0.78 & $0.45-1.33$ & 0.36 \\
Age & 1.02 & $1.00-1.04$ & 0.5
\end{tabular}

Treatment (Surgery + Chemoradiation reference)

\begin{tabular}{lccc} 
Surgery + RT & 1.31 & $0.63-2.73$ & 0.461 \\
Chemoradiation & 3.51 & $1.76-6.99$ & $<0.0001$ \\
Radiation & 8.46 & $4.07-15.80$ & $<0.0001$ \\
\hline
\end{tabular}

$\mathrm{HR}=$ hazard ratio. $\mathrm{Cl}=$ confidence interval.

$21 \mathrm{HPV}$ positive oropharyngeal cancer specimens were also basaloid differentiated. Mendelsohn et al. [11] found that 8 of 17 p16 positive and only 4 of $11 \mathrm{HPV}$ positive by in situ hybridization head and neck cancers exhibited basaloid differentiation. Similarly, Gillison et al. [3] found that HPV positive oropharyngeal cancer was more likely to be basaloid differentiated. Basaloid differentiation was found in 11 of $34 \mathrm{HPV}$ positive oropharyngeal tumors. However, about one third of tumor specimens did not have data available on basaloid differentiation in this study. Also, Hafkamp et al. [12] reported that 4 of 9 HPV positive oropharyngeal cancer specimens showed basaloid differentiation. Previously reported evidence of an association between basaloid differentiation and p16 positivity in oropharyngeal cancer consists primarily of small studies, some of which were not specific to the oropharynx. Our series is the largest to date to quantify basaloid differentiation in oropharyngeal cancer and its relation to p16 status.

We found basaloid differentiation to be a significant positive prognostic indicator in oropharyngeal squamous cell carcinoma. Patients with basaloid differentiated tumors had a 33\% greater 5 year DSS compared to nonbasaloid tumors. This finding has not previously been shown in a large series of patients. The positive prognostic implications of p16 have been well documented $[10,15,18,19]$. The magnitude of survival advantage with basaloid differentiation was comparable to that of p16 positivity in this study. Patients who were p16 positive had a $38 \%$ greater 5 year DSS compared to p16 negative patients. Based on these results, comparable survival prognostication could be obtained from either basaloid differentiation or p16 IHC. This has immediate practical applications to regions of the world that do not have access to p16 IHC or other HPV-related assays.

Further prognostic information is provided by combining basaloid differentiation with p16 status. Patients with basaloid differentiated, p16 positive tumors had the best survival outcomes, followed by non-basaloid and p16 positive tumors, then basaloid and p16 negative tumors, and lastly non-basaloid and p16 negative tumors. Due to the added prognostic value of basaloid differentiation, we are of the opinion that this feature should be routinely reported by qualified pathologists.

There were some limitations in this study. The retrospective nature of the study means that not all factors such as Eastern Cooperative Oncology Group (ECOG)

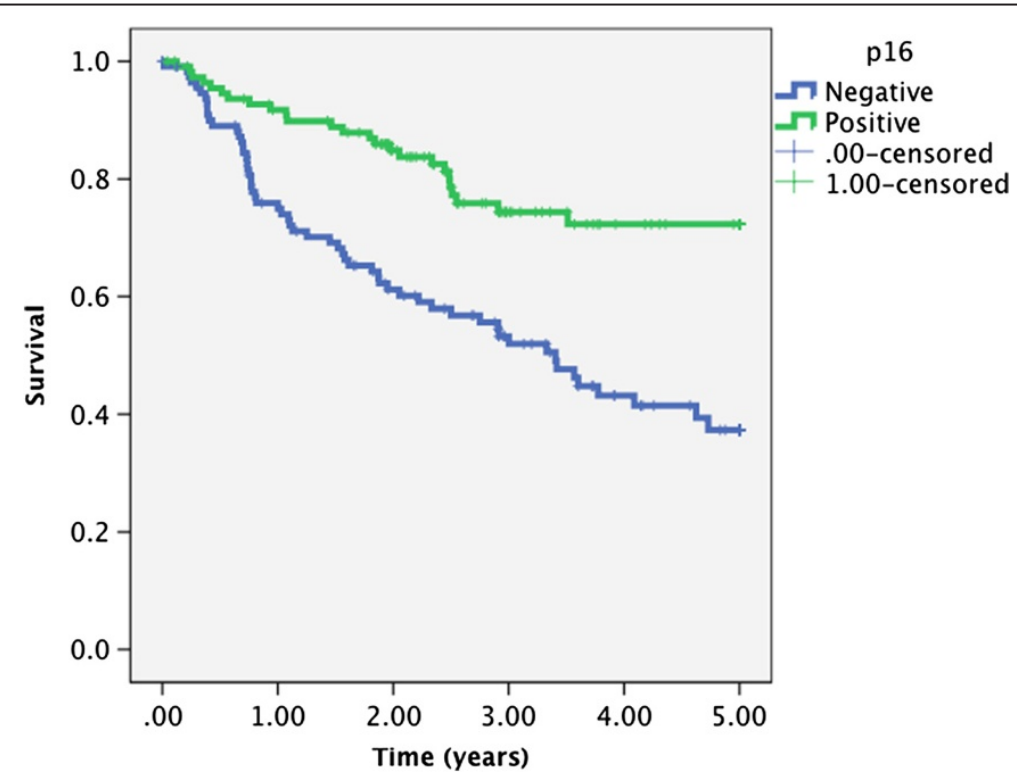

Figure 1 Kaplan-Meier analysis of disease specific survival in 208 patients according to $p 16$ status $(p<0.001$, log rank test). 


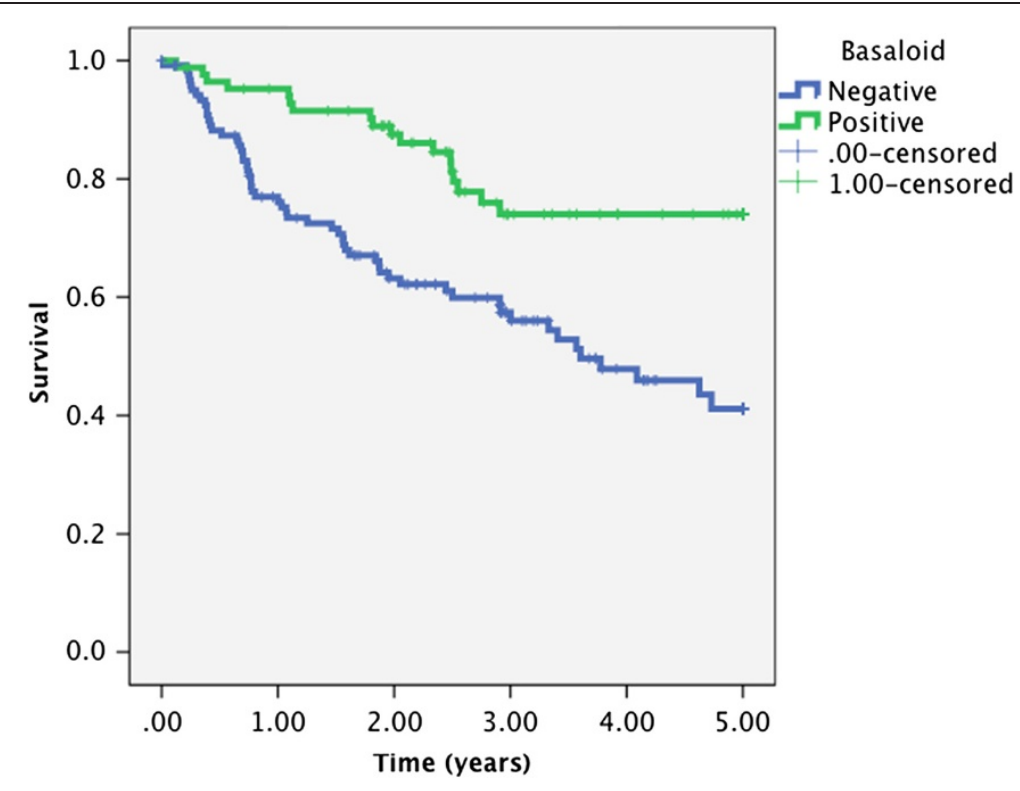

Figure 2 Kaplan-Meier analysis of disease specific survival in 208 patients according to basaloid differentiation ( $p=0.001$, log rank test).

Performance Status [20] and Charlson Comorbidity Index (CCI) [21] were taken into account. In addition, smoking status and alcohol consumption data were unreliable and thus not included in our multivariate analysis. There is, therefore, potential for bias within the groups, which may impact outcomes. In addition, the treatment modalities used were not equally distributed between groups and this could be another confounding factor beyond our control. One possible explanation for this is that basaloid differentiated and p16 positive patients were more likely to have nodal disease and may have been treated more aggressively with surgery followed

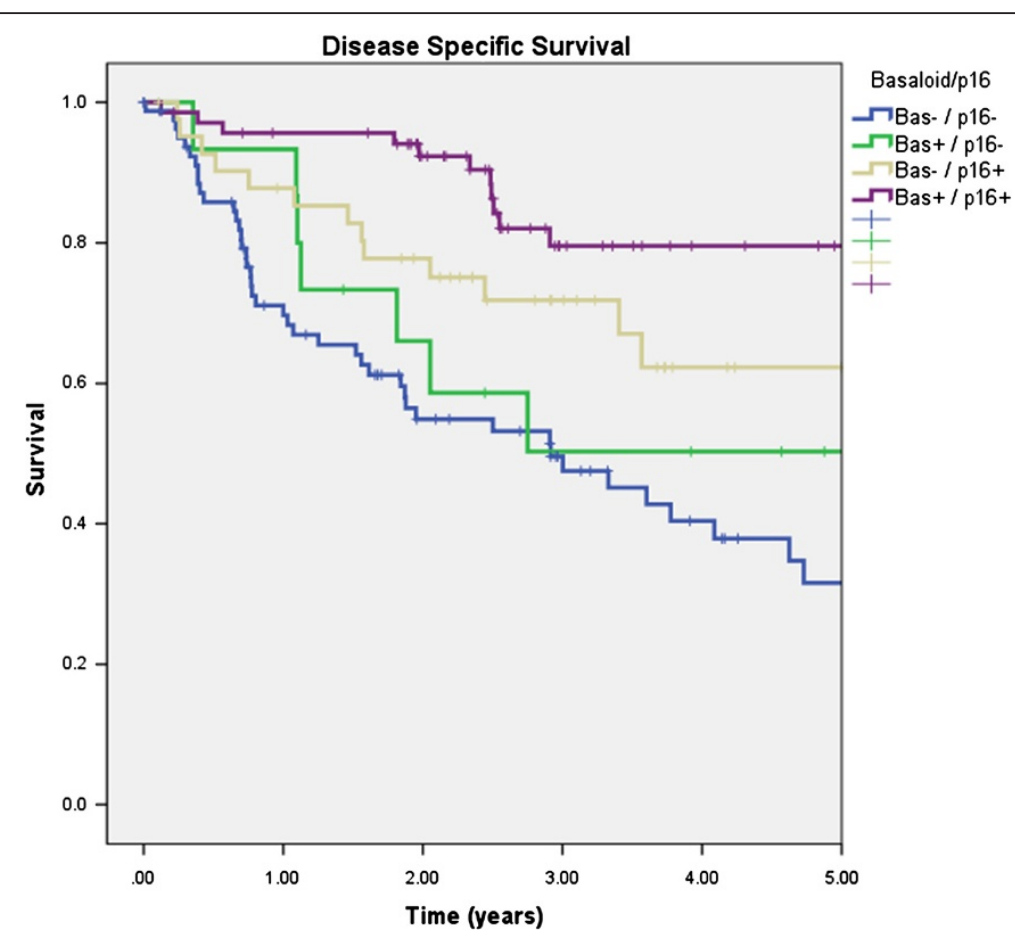

Figure 3 Kaplan-Meier analysis of disease specific survival in 208 patients according to basaloid differentiation and p16 status $(\mathbf{p}<0.001$, log rank test). Bas- = non-basaloid. Bas $+=$ basaloid differentiated. 
by chemoradiation for this reason. All patients were identified from and treated at a single institution and although our institution is responsible for treatment of all oropharyngeal cancer within the region, our outcomes and treatment modalities may not be representative of other centers. Some patients were not able to be included in the study due to inadequate tissue availability for analysis. We were unable to perform real-time PCR analysis for HPV which would provide additional HPV status information. This would involve RNA isolation from the tissue samples and cDNA amplification which was not possible with the FFPE specimens used in this study.

\section{Conclusions}

Risk stratification and prognostication based on tumor molecular characteristics is rapidly expanding in head and neck cancer. The focus has been on adding additional significant molecular markers to the array in order to gain further prognostic information and guide management. There is additional value and information to be obtained from a process that is already routinely performed, H\&E staining. Basaloid differentiation in oropharyngeal cancer is an easy and inexpensive predictor of survival. The significant survival advantage based on basaloid differentiation alone is a novel finding for which the molecular basis should be further explored.

\section{Abbreviations}

CCI: Charlson Comorbidity Index; DSS: Disease specific survival; ECOG: Eastern Cooperative Oncology Group; FFPE: Formalin-fixed paraffin-embedded; HPV: Human papillomavirus; H\&E: Hematoxylin and eosin;

IHC: Immunohistochemistry; PCR: Polymerase chain reaction; RT-PCR: Reverse transcriptase polymerase chain reaction; TMA: Tissue microarray.

\section{Competing interests}

The authors have no financial or non-financial competing interests to disclose.

\section{Authors' contributions}

TC contributed with data collection, data analysis, writing and revision of the manuscript. VB contributed with conception and design, data collection, data analysis, and critical revision of the manuscript. BA contributed with data collection and data analysis. AK contributed with TMA construction and optimization of p16 staining and quantification. LP contributed with conception and design, data collection, and critical revision of the manuscript. HS contributed with conception and design, data collection, data analysis, writing and revision of the manuscript. All authors have given final approval of the version to be published.

\section{Acknowledgements}

We would like to acknowledge Mie Konno and Michelle Dean from the Tom Baker Cancer Center for TMA construction and AQUAnalysis. Also, Val Taylor and Steve Joy from the University of Alberta Department of Pathology for tissue specimen acquisition. Thank-you to Dr. David Côté from the Division of Otolaryngology-Head and Neck Surgery at the University of Alberta for assistance with critical revision of the manuscript.

\section{Author details}

${ }^{1}$ Division of Otolaryngology - Head and Neck Surgery, Department of Surgery, University of Alberta, 1E4 University of Alberta Hospital, Edmonton, AB T6G 2B7, Canada. ${ }^{2}$ Department of Laboratory Medicine \& Pathology, University of Alberta, 5B2 University of Alberta Hospital, Edmonton, AB T6G
2B7, Canada. ${ }^{3}$ Department of Oncology, University of Calgary, Tom Baker Cancer Centre, 1331-29 Street NW, Calgary, AB T2N 4 N2, Canada.

Received: 2 April 2013 Accepted: 21 November 2013 Published: 19 December 2013

\section{References}

1. Cardesa A, Nadal A: Carcinoma of the head and neck in the HPV era. Acta Dermatovenerol Alp Panonica Adriat 2011, 20(3):161-173.

2. El-Mofty SK, Patil S: Human papillomavirus (HPV)-related oropharyngeal nonkeratinizing squamous cell carcinoma: characterization of a distinct phenotype. Oral Surg Oral Med Oral Pathol Oral Radiol Endod 2006, 101(3):339-345.

3. Gillison ML, Koch WM, Capone RB, et al: Evidence for a causal association between human papillomavirus and a subset of head and neck cancers. J Natl Cancer Inst 2000, 92(9):709-720.

4. Klussmann JP, Weissenborn SJ, Wieland U, et al: Prevalence, distribution, and viral load of human papillomavirus 16 DNA in tonsillar carcinomas. Cancer 2001, 92(11):2875-2884.

5. El-Mofty SK, Lu DW: Prevalence of human papillomavirus type 16 DNA in squamous cell carcinoma of the palatine tonsil, and not the oral cavity, in young patients: a distinct clinicopathologic and molecular disease entity. Am J Surg Pathol 2003, 27(11):1463-1470.

6. Nichols AC, Faquin WC, Westra WH, et al: HPV-16 infection predicts treatment outcome in oropharyngeal squamous cell carcinoma. Otolaryngol Head Neck Surg 2009, 140(2):228-234.

7. Westra WH: The changing face of head and neck cancer in the $21 \mathrm{st}$ century: the impact of HPV on the epidemiology and pathology of oral cancer. Head Neck Pathol 2009, 3(1):78-81.

8. Schache AG, Liloglou T, Risk JM, et al: Evaluation of human papilloma virus diagnostic testing in oropharyngeal squamous cell carcinoma: Sensitivity, specificity, and prognostic discrimination. Clin Cancer Res 2011, 17(19):6262-6271.

9. Dufour X, Beby-Defaux A, Agius G, Lacau St Guily J: HPV and head and neck cancer. Eur Ann Otorhinolaryngol Head Neck Dis 2012, 129(1):26-31.

10. Lau HY, Brar S, Klimowicz AC, et al: Prognostic significance of p16 in locally advanced squamous cell carcinoma of the head and neck treated with concurrent cisplatin and radiotherapy. Head Neck 2011, 33(2):251-256

11. Mendelsohn AH, Lai CK, Shintaku IP, et al: Histopathologic findings of HPV and p16 positive HNSCC. Laryngoscope 2010, 120(9):1788-1794.

12. Hafkamp HC, Speel EJ, Haesevoets A, et al: A subset of head and neck squamous cell carcinomas exhibits integration of HPV 16/18 DNA and overexpression of p16INK4A and p53 in the absence of mutations in p53 exons 5-8. Int J Cancer 2003, 107(3):394-400.

13. Chernock RD, El-Mofty SK, Thorstad WL, Parvin CA, Lewis JS Jr: HPV-related nonkeratinizing squamous cell carcinoma of the oropharynx: utility of microscopic features in predicting patient outcome. Head Neck Pathol 2009, 3(3):186-194

14. Wilczynski SP, Lin BT, Xie Y, Paz IB: Detection of human papillomavirus DNA and oncoprotein overexpression are associated with distinct morphological patterns of tonsillar squamous cell carcinoma. Am J Pathol 1998, 152(1):145-156.

15. Laco J, Nekvindova J, Novakova V, et al: Biologic importance and prognostic significance of selected clinicopathological parameters in patients with oral and oropharyngeal squamous cell carcinoma, with emphasis on smoking, protein p16(INK4a) expression, and HPV status. Neoplasma 2012, 59(4):398-408.

16. Klimowicz AC, Bose P, Nakoneshny SC, et al: Basal Ki67 expression measured by digital image analysis is optimal for prognostication in oral squamous cell carcinoma. Eur J Cancer 2012, 48(14):2166-2174.

17. Hong AM, Martin A, Armstrong BK, et al: Human papillomavirus modifies the prognostic significance of $\mathrm{T}$ stage and possibly $\mathrm{N}$ stage in tonsillar cancer. Ann Oncol 2013, 24(1):215-219.

18. Lewis JS Jr, Thorstad WL, Chernock RD, et al: p16 Positive oropharyngeal squamous cell carcinoma: an entity with a favorable prognosis regardless of tumor HPV status. Am J Surg Pathol 2010, 34(8):1088-1096.

19. Fakhry C, Westra WH, Li S, et al: Improved survival of patients with human papillomavirus-positive head and neck squamous cell carcinoma in a prospective clinical trial. J Natl Cancer Inst 2008, 100(4):261-269. 
20. Oken MM, Creech RH, Tormey DC, et al: Toxicity and response criteria of the eastern cooperative oncology group. Am J Clin Oncol 1982, 5(6):649-655.

21. Charlson ME, Pompei P, Ales KL, Mackenzie CR: A new method of classifying prognostic comorbidity in longitudinal studies: development and validation. J Chronic Dis 1987, 40(5):373-383.

doi:10.1186/1916-0216-42-57

Cite this article as: Cooper et al:: Prognostic utility of basaloid

differentiation in oropharyngeal cancer. Journal of Otolaryngology - Head and Neck Surgery 2013 42:57.

\section{Submit your next manuscript to BioMed Central and take full advantage of:}

- Convenient online submission

- Thorough peer review

- No space constraints or color figure charges

- Immediate publication on acceptance

- Inclusion in PubMed, CAS, Scopus and Google Scholar

- Research which is freely available for redistribution 